\title{
On the dispersion in lithium and potassium among late-type stars in young clusters: IC 2602*
}

\author{
S. Randich \\ Osservatorio Astrofisico di Arcetri, Largo Fermi 5, 50125 Firenze, Italy \\ Received 23 January 2001 / Accepted 23 July 2001

\begin{abstract}
We have measured the equivalent width $(E W)$ of the $\mathrm{K}$ I $7699 \AA$ line in a sample of $\mathrm{G}$ and $\mathrm{K}$-type members of the $\sim 35 \mathrm{Myr}$ old cluster IC 2602 for which a dispersion in Li $E W \mathrm{~s}$ had been reported by previous studies. Active cluster stars with $0.75 \lesssim B-V_{0} \lesssim 1$ are characterized by a dispersion in the $E W$ of the K I $7699 \AA$, while earlier and later-type stars do not show any significant scatter. Cluster stars at all colors show potassium $E W$ excesses with respect to field inactive stars; furthermore, a statistically significant relationship is found between differential potassium $E W \mathrm{~s}$ and $\log L_{\mathrm{X}} / L_{\mathrm{bol}}$ ratios, indicating that the $E W \mathrm{~s}$ of the potassium feature are altered by activity. Our results suggest that the dispersion in Li $E W$ s observed among cluster stars later than $B-V_{0} \sim 1$ cannot be fully explained by the effects of activity. No final conclusion can instead be drawn for earlier-type stars.
\end{abstract}

Key words. open clusters and associations: individual: IC 2602 - stars: abundances - stars: interiors

\section{Introduction}

The existence of a star-to-star scatter in $\mathrm{Li}$ abundances among otherwise similar late-type members of the 120 Myr old Pleiades cluster was first reported by Duncan \& Jones (1983) and Butler et al. (1987); the dispersion was subsequently confirmed by several studies and additional observational constraints were put on it based on larger samples (Soderblom et al. 1993a; García López et al. 1994; Jones et al. 1996). A star-to-star scatter in Li has also been reported for other clusters both younger and older than the Pleiades, such as Alpha Per (Balachandran et al. 1996; Randich et al. 1998), IC 2602 (Randich et al. 1997 - hereafter R97; Randich et al. 2001 - hereafter R01), IC 4665 (Martín \& Montes 1997), M 34 (Jones et al. 1997), NGC 6475 (James \& Jeffries 1997; James et al. 2000; Randich et al. 2000). The scatter has disappeared by the Hyades age (600 Myr). The detection of a dispersion is one of the most puzzling results within the context of the so-called Li problem (e.g. Jeffries 2000 and references therein), being in strong contradiction with the predictions of "standard models" of stellar evolution; standard models incorporate convection only as a mixing mechanism and predict that the amount of Li depletion should

\footnotetext{
Send offprint requests to: S. Randich,

e-mail: randich@arcetri.astro.it

* Based on observations carried out at the European Southern Observatory, La Silla, Chile.
}

depend on mass, age, and metallicity (or chemical composition) only; no differences in $\mathrm{Li}$ abundances are indeed expected for co-eval, otherwise similar stars in clusters. We mention in passing that the spread in Li in the Pleiades was initially ascribed to a large spread in age; Soderblom et al. (1993a, 1993b), however, convincingly showed that this is unlikely the case. Also note that the hypothesis that errors in stellar parameters, in particular effective temperature, might be a source of dispersion is rather unlikely, since a large scatter is present in the $E W$ vs. color diagrams, i.e., stars with the same color (and presumably mass) in the same cluster have different Li $E W \mathrm{~s}$.

Under the assumption that the observed scatter in $\mathrm{Li}$ reflects a real scatter in abundances, much work has been done on theoretical grounds in order to explain it; extramixing processes and/or mechanisms able to inhibit $\mathrm{Li}$ destruction were introduced in the models. Driven by the observed Li-rotation relationship, most of the models included rotation and/or angular momentum loss as an additional crucial parameter determining the amount of $\mathrm{Li}$ depletion (e.g., Martín \& Claret 1996; Pinsonneault 1997 and references therein): according to these models, different rotation rates or rotational histories would lead to different Li abundances. None of the models so far elaborated is able to quantitatively explain the observed features.

In the last few years there has been a growing interest in investigating whether and to what extent the observed scatter in the $E W \mathrm{~s}$ of the $\mathrm{Li}$ I $6708 \AA$ resonance doublet 
among young cluster stars truly reflects a dispersion in abundances or is rather due to other effects which could affect the formation of the Li line. More specifically, the suggestion has been made that chromospheric activity (including the presence of chromospheres as well as surface inhomogeneities such as spots and plages) may affect the formation of the $\mathrm{Li}$ line and be the reason for the observed spread in $E W \mathrm{~s}$, since, for a given abundance and temperature, more active stars would have larger $E W \mathrm{~s}$. In this case, the dispersion would not witness a dispersion in abundances and the high-Li - high-rotation relationship would not be a direct relationship, but it would be the consequence of the fact that high rotators are characterized by high activity levels and thus their Li $E W$ s are more affected by activity.

Various ways of addressing this issue exist: we focus here on the simultaneous measurement of the Li I $6708 \AA$ and the K I $7699 \AA$ resonance features. The excitation potential of the potassium line and, more in general, its formation conditions, are very similar to those of the $\mathrm{Li}$ doublet; any line formation effect that alters the Li line should also affect the $\mathrm{K}$ I line and viceversa. Potassium is not destroyed in stars and star-to-star differences in its abundance among members of the same cluster are not expected: therefore, the detection of a spread in the $E W$ of the K I line may provide an indication that the scatter in Li $E W$ s does not necessarily imply a scatter in abundances.

The effect of activity on the Li I and K I lines has been quantitatively studied by Stuik et al. (1997), who modeled the atmospheric stratifications of various combinations of spots and plages and investigated how those stratifications affect lithium and potassium line formation. They showed that the two alkali lines are not sensitive to chromospheres, but they are affected by the presence of plages and spots, which also significantly alter broad-band stellar colors and thus the observed alkali $E W$ vs. color diagrams. Stuik et al. were not able to reproduce the Pleiades observational pattern and concluded that, whereas it is not easy to demonstrate that the dispersion in $\mathrm{K}$ I $E W \mathrm{~s}$ (and Li I $E W \mathrm{~s}$ ) is really and completely due to stellar activity, the presence of a scatter in potassium $E W$ s constitutes a warning against interpreting the spread in $\mathrm{Li}$ as due to a real spread in abundances.

A few additional studies on this topic were recently carried out: Jeffries (1999) using new data and old data from the literature, simultaneously monitored the strengths of the Li I and $\mathrm{K}$ I lines and $\mathrm{H} \alpha$ in a sample of Pleiades K-type stars to search for variability of the line strengths which would witness the presence of large-scale atmospheric inhomogeneities. He detected no variability of $\mathrm{Li} \mathrm{I} E W \mathrm{~s}$ on one year timescales, possible variability on 10 years timescales, and only $20-30 \%$ variability in chromospheric activity; he confirmed the presence of a dispersion in potassium and a correlation between $\mathrm{Li}$ and $\mathrm{K}$ line strengths and rotation and activity. Similarly to Stuik et al. (1997), he concluded that the dispersion in $\mathrm{K}$ I $E W \mathrm{~s}$ must be explained before definitively accepting that the dispersion in $\mathrm{Li}$ is due to a genuine dispersion in abundances. King et al. (2000) instead, based on a new analysis of Pleiades data from the literature, found that an excess in Li abundance correlates with an excess in the potassium $E W$ and activity and concluded, more firmly than the other studies, that activity is, at least in part, the reason for the dispersion in Li $E W$ s. Finally, Barrado y Navascués et al. (2001) presented an analytical model to investigate the effect of stellar surface inhomogeneities on the Li I and K I features (plus the Na I $5896 \AA$ feature) and compared their predicted $E W \mathrm{~s}$ with the observed $E W \mathrm{~s}$ in the Pleiades. They concluded, that activity can explain part of the dispersion, but it cannot fully account for it. The issue therefore is far from being settled.

So far, no studies of the potassium feature among latetype stars in other young clusters have been carried out. In this paper we present new potassium data for the $35 \mathrm{Myr}$ old IC 2602; as mentioned above, a dispersion in Li was detected among its late-type members by R97 and R01, although the scatter seems narrower than in the Pleiades. With the present study we wish to address the obvious questions whether IC 2602 stars are also characterized by a dispersion in $\mathrm{K}$ I $E W \mathrm{~s}$ and whether there is a correlation between potassium $E W$ s and activity. A positive answer to these questions would provide an additional hint that activity do affect the formation of the alkali lines.

\section{Observational data}

The equivalent widths of the K I $7699 \AA$ line were measured in a sample of IC 2602 stars selected from R97 and R01. Ca I $6718 \AA$ line strengths were also measured for the sample stars. Most of the sample stars had been observed using CASPEC at the ESO $3.6 \mathrm{~m}$ telescope; high resolution spectra for a few of them were acquired using the Echelle spectrograph on the CTIO $4 \mathrm{~m}$ telescope. We refer to R97, R01, and Stauffer et al. (1997) for details on the observations; we briefly recall here that the resolving powers ranged between $R \sim 20000$ and $R \sim 41000$, due to the use of different instruments and/or slit widths. $S / N$ ratios in the $\mathrm{K}$ I line spectral region are in the range between 60 and 100, although a few spectra were characterized by lower $S / N$ ratios, which did not allow us to measure their potassium features. The present sample includes all the stars with $B-V_{0} \leq 1.4$ for which we were able to get a reliable measurement of the $E W$ of the $\mathrm{K}$ I feature; $E W \mathrm{~s}$ were measured, as usual, by direct integration below the continuum levels. These in turn were estimated by polynomial fitting of line free regions. The sample stars are listed in Table 1. Star names come from Randich et al. (1995) (with exception of W79 - from Whiteoak 1961); in Cols. 2 and 3 we list $B-V_{0}$ and $V-I_{0}$ colors which were retrieved from Prosser et al. (1996); as in R01 reddening values $E(B-V)=E(V-I)=0.04$ were assumed. The measured $E W$ s of the Li I (retrieved from R97 and R01), $\mathrm{K}$ I, and Ca I lines, together with $1 \sigma$ errors, are listed in Cols. 4-6. 
Table 1. Sample stars and measured equivalent widths.

\begin{tabular}{|c|c|c|c|c|c|}
\hline star & $B-V_{0}$ & $V-I_{0}$ & $\begin{array}{c}E W(\operatorname{Li~I~} \lambda 6708 \AA) \\
(\mathrm{m} \AA)\end{array}$ & $\begin{array}{c}E W(\mathrm{~K} \text { I } \lambda 7699 \AA) \\
(\mathrm{m} \AA)\end{array}$ & $\begin{array}{c}E W(\mathrm{Ca} \mathrm{I} \lambda 6718 \AA) \\
(\mathrm{m} \AA)\end{array}$ \\
\hline W 79 & 0.79 & 0.81 & $142 \pm 5$ & $214 \pm 11$ & $158 \pm 9$ \\
\hline R 1 & 0.87 & 0.87 & $204 \pm 7$ & $324 \pm 15$ & $176 \pm 12$ \\
\hline R 3 & 0.83 & 0.85 & $265 \pm 13$ & $269 \pm 15$ & $151 \pm 9$ \\
\hline R 7 & 0.40 & 0.42 & $65 \pm 17$ & - & $71 \pm 25$ \\
\hline R 8 & 0.61 & 0.62 & $178 \pm 9$ & $160 \pm 10$ & $122 \pm 6$ \\
\hline R 14 & 0.83 & 0.84 & $230 \pm 10$ & $265 \pm 12$ & $177 \pm 10$ \\
\hline R 15 & 0.89 & 1.02 & $255 \pm 15$ & $325 \pm 15$ & $180 \pm 12$ \\
\hline R 21 & 0.47 & 0.58 & $119 \pm 10$ & $97 \pm 15$ & $110 \pm 10$ \\
\hline R 29 & 1.07 & 1.15 & $239 \pm 11$ & $385 \pm 14$ & $237 \pm 10$ \\
\hline R 35 & 0.63 & 0.66 & $215 \pm 8$ & $160 \pm 20$ & $138 \pm 9$ \\
\hline R 43 & 0.95 & 1.10 & $355 \pm 20$ & $400 \pm 20$ & $193 \pm 20$ \\
\hline R 45 & 0.62 & 0.68 & $151 \pm 7$ & $160 \pm 10$ & $124 \pm 5$ \\
\hline R 54 & 1.11 & 1.33 & $189 \pm 10$ & $425 \pm 20$ & $230 \pm 20$ \\
\hline R 58 & 0.61 & 0.70 & $204 \pm 27$ & - & $108 \pm 30$ \\
\hline R 59 & 0.78 & 0.96 & $305 \pm 15$ & $310 \pm 15$ & $159 \pm 10$ \\
\hline R 66 & 0.64 & 0.79 & $173 \pm 10$ & $215 \pm 10$ & $135 \pm 8$ \\
\hline R 68 & 0.82 & 1.05 & $266 \pm 10$ & $380 \pm 10$ & $170 \pm 10$ \\
\hline R 70 & 0.64 & 0.67 & $172 \pm 12$ & $165 \pm 5$ & $139 \pm 7$ \\
\hline R 72 & 0.62 & 0.72 & $209 \pm 15$ & $210 \pm 20$ & $141 \pm 15$ \\
\hline R 79 & 0.40 & 0.47 & $61 \pm 20$ & - & $50 \pm 15$ \\
\hline R 85 & 0.48 & 0.54 & $103 \pm 10$ & $90 \pm 10$ & $66 \pm 10$ \\
\hline R 89 & 1.20 & 1.31 & $265 \pm 8$ & $425 \pm 15$ & $260 \pm 10$ \\
\hline R 92 & 0.65 & 0.74 & $195 \pm 10$ & $210 \pm 5$ & $147 \pm 6$ \\
\hline R 93 & 1.33 & 1.58 & $60 \pm 10$ & $547 \pm 17$ & $303 \pm 17$ \\
\hline R 94 & 1.35 & 1.69 & $174 \pm 8$ & $535 \pm 14$ & $313 \pm 15$ \\
\hline R 95 & 0.83 & 0.93 & $317 \pm 15$ & $350 \pm 10$ & $184 \pm 7$ \\
\hline R 96 & 1.21 & 1.33 & $320 \pm 12$ & $469 \pm 18$ & $251 \pm 11$ \\
\hline
\end{tabular}

\section{Results}

\subsection{The dispersion in the EW vs. color diagrams}

In Fig. 1 we plot the equivalent width of the potassium line vs. $B-V_{0}$ (left-hand panel) and $V-I_{0}$ (right-hand panel) colors for IC 2602 members included in the present sample; Figs. 2 and 3 are the same as Fig. 1, but the $E W \mathrm{~s}$ of the $\mathrm{Li}$ I and $\mathrm{Ca}$ I $6718 \AA$ lines are shown, respectively. It is worthwhile recalling that calcium is not an alkaline element; however, since the Ca I $6718 \AA$ is not thought to be affected by activity, it is used here for comparison purposes.

The comparison of the three figures first shows that, whereas the $E W \mathrm{~s}$ of the $\mathrm{K} \mathrm{I}$ and $\mathrm{Ca}$ I lines monotonically increase with color, as well known, this is not the case for lithium. The difference in the $E W$ vs. color morphologies of the three lines is due to the fact that, as mentioned in Sect. 1 (and this is indeed the motivation for the present and similar studies), lithium is destroyed in low mass stars, with the amount of depletion increasing with decreasing mass, while potassium and calcium are not; therefore, the
$E W$ s of the $\mathrm{Ca}$ I and $\mathrm{K}$ I lines are determined by effective temperature only, since we are considering stars in the same evolutionary status, and thus with similar gravities and microturbulence values; on the contrary, the strength of the $\mathrm{Li}$ line is determined by both the effective temperature (for a fixed abundance the $E W$ would increase with decreasing temperature) and the abundance, which decreases towards cooler stars. The $E W(\mathrm{Li})$ vs. color morphology for cluster stars is indeed a very well known result and a more detailed discussion is not warranted here. It is instead important to stress again that, according to standard models, at a given color or effective temperature, stars in the same cluster should have the same Li abundance, and thus Li $E W$, and a tight Li vs. color distribution should be observed.

Figures 1-3 also suggest that: i) as already pointed out by $\mathrm{R} 97$ and R01, a dispersion in the $E W$ of the $\mathrm{Li}$ line is present among cluster stars, i.e., stars with similar colors have different $E W \mathrm{~s}$; ii) A dispersion in potassium $E W \mathrm{~s}$ seems also to be present in the $E W$ vs. $B-V$ diagram; the dispersion seems narrower when looking at the 

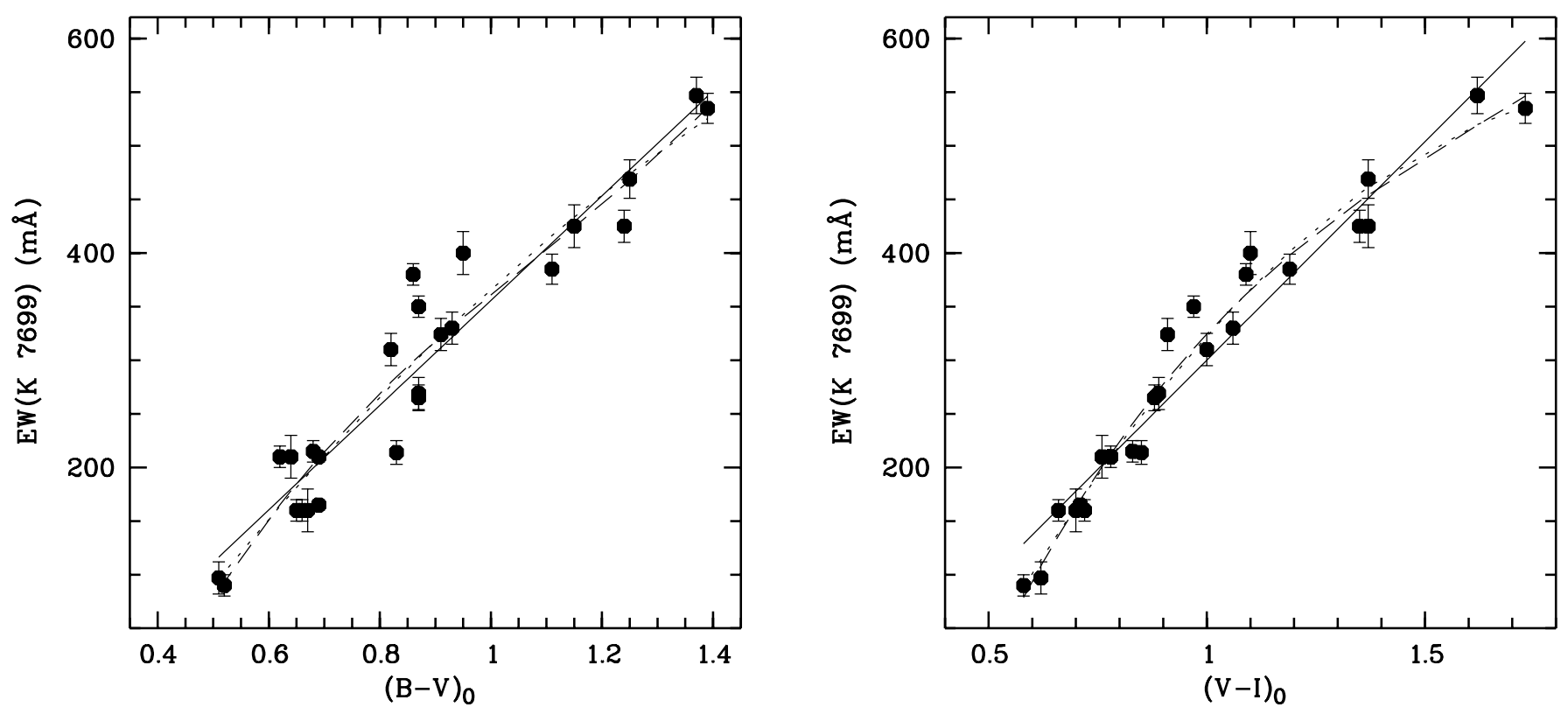

Fig. 1. K I $7699 \AA$ equivalent width vs. dereddened stellar color $\left(B-V_{0}\right.$ and $V-I_{0}$ in the left- and right-hand panels, respectively) for our sample stars. The three curves indicate polynomial regressions of grade 1 (solid), 2 (dotted), 3 (dashed).
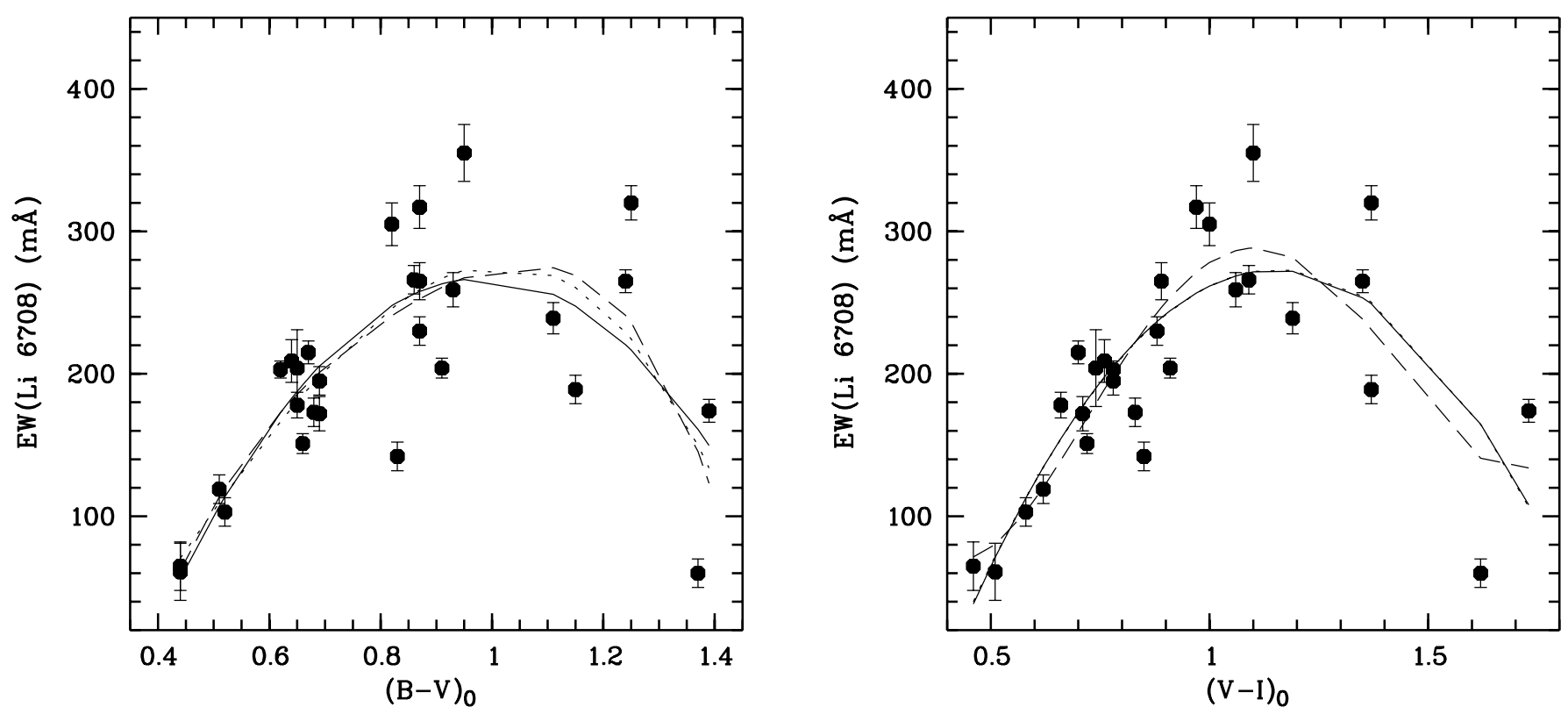

Fig. 2. Same as Fig. 1, but the equivalent width of the Li I $6708 \AA$ line is plotted as a function of the two stellar colors. Grade 2 (solid), 3 (dotted), and 4 (dashed) polynomial regressions were carried out for this line.

$E W$ vs. $V-I$ diagram; iii) The dispersion is most evident for stars with $0.8 \lesssim B-V_{0} \lesssim 1$; on the contrary, late-K stars $\left(B-V_{0} \gtrsim 1\right)$ do not show a large spread in potassium $E W \mathrm{~s}$, although they do show a dispersion in Li (see Fig. 2); iv) Finally, no significant (i.e., larger than measurement errors) dispersion seems to be present in the calcium $E W$ vs. color diagrams.

We carried out a more quantitative analysis by performing polynomial regressions of the observed $E W$ vs. color distributions and estimating reduced $\chi^{2}$ values that allow assessing on a statistical basis the presence/lack of a star-to-star scatter. The reduced $\chi^{2}$ values for the three atoms as a function of the two colors and for different $B-V$ ranges are listed in Table 2 . In the table we also provide the probability that the observed dispersions are real. The symbol "S" means a probability larger than $99.9 \%$ (i.e., a significance level larger than $3 \sigma$ ), while "NS" means a probability below $67 \%$ (i.e., below $1 \sigma$ significance). The regression curves are also shown in the three figures. Note that the polynomial regressions we have carried out do not have any real physical meaning (i.e., we are not trying to model the $E W$ vs. color patterns); our aim here is to 

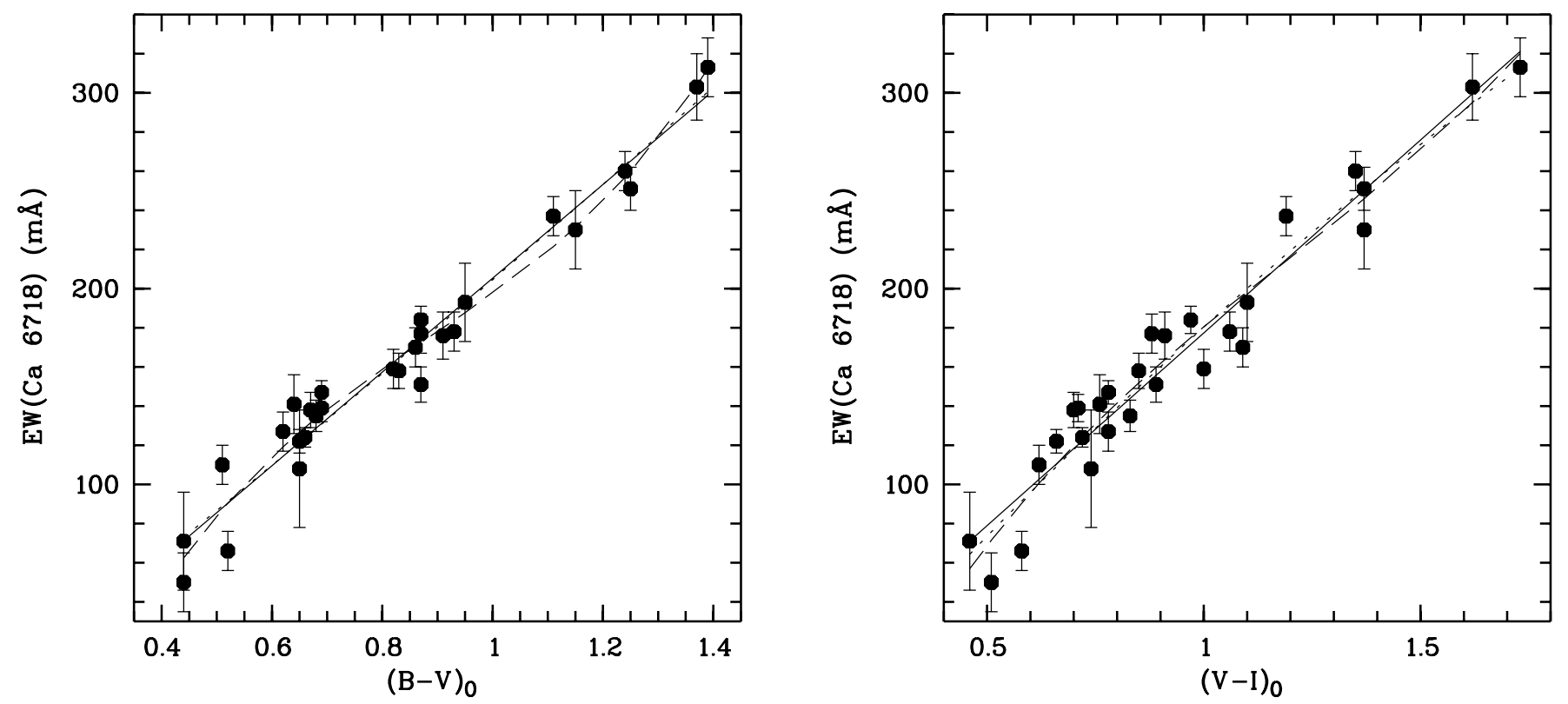

Fig. 3. Same as Fig. 1, but the $E W$ s of the Ca I $6718 \AA$ line are shown.

Table 2. The scatter in the $E W$ vs. color diagrams: reduced $\chi^{2}$ values and probabilities that the dispersion is real (numbers within parenthesis) are listed. "S" means a probability larger than $99.9 \%$, while "NS" indicates a probability below $67 \%$. "Degree" indicates the degree of the polynomial fitting of the $E W$ vs. color relationship (see text for details). "All" refers to the whole color range, while intervals $\mathrm{A}$, $\mathrm{B}$, and $\mathrm{C}$, refer to $B-V_{0}<0.75,0.75 \leq B-V_{0} \leq 1$, and $B-V_{0}>1$, respectively.

\begin{tabular}{|c|cccc|ccccc|c|}
\hline \hline color/degree & \multicolumn{4}{|c|}{ Li I $(6708 \AA)$} & \multicolumn{4}{c|}{ K I $(7699 \AA)$} & Ca I (6718 A) \\
& All & A & B & C & All & A & B & C & All \\
\hline$B-V / 1$ & - & - & - & - & $12.6(\mathrm{NS})$ & $11.3(\mathrm{NS})$ & $19.6(98 \%)$ & $2.4(\mathrm{NS})$ & $1.5(\mathrm{NS})$ \\
$B-V / 2$ & $36.1(91 \%)$ & $6.9(\mathrm{NS})$ & $66(\mathrm{~S})$ & $42(\mathrm{~S})$ & $12.2(\mathrm{NS})$ & $10.7(\mathrm{NS})$ & $17.4(96 \%)$ & $2.9(\mathrm{NS})$ & $1.6(\mathrm{NS})$ \\
$B-V / 3$ & $37.2(94 \%)$ & $6.8(\mathrm{NS})$ & $66(\mathrm{~S})$ & $41(\mathrm{~S})$ & $13.1(\mathrm{NS})$ & $12.1(72 \%)$ & $17.5(96 \%)$ & $1.8(\mathrm{NS})$ & $1.3(\mathrm{NS})$ \\
$B-V / 4$ & $36.2(95 \%)$ & $6.3(\mathrm{NS})$ & $60(\mathrm{~S})$ & $41(\mathrm{~S})$ & - & - & - & - & - \\
\hline$V-I / 1$ & - & - & - & - & $6.7(\mathrm{NS})$ & $4.7(\mathrm{NS})$ & $4.9(\mathrm{NS})$ & $4.1(\mathrm{NS})$ & $2.5(\mathrm{NS})$ \\
$V-I / 2$ & $27.8(\mathrm{NS})$ & $6.6(\mathrm{NS})$ & $42(\mathrm{~S})$ & $43(\mathrm{~S})$ & $3.1(\mathrm{NS})$ & $1.4(\mathrm{NS})$ & $1.3(\mathrm{NS})$ & $1.6(\mathrm{NS})$ & $2.4(\mathrm{NS})$ \\
$V-I / 3$ & $28.9(73 \%)$ & $6.5(\mathrm{NS})$ & $41(\mathrm{~S})$ & $44(\mathrm{~S})$ & $3.2(\mathrm{NS})$ & $1.8(\mathrm{NS})$ & $1.3(\mathrm{NS})$ & $1.6(\mathrm{NS})$ & $2.4(\mathrm{NS})$ \\
$V-I / 4$ & $28.9(77 \%)$ & $7.9(\mathrm{NS})$ & $50(\mathrm{~S})$ & $31(\mathrm{~S})$ & - & - & - & - & - \\
\hline \hline
\end{tabular}

infer a "mean" $E W$ as a function of color and to ascertain whether the scatter around this mean $E W$ is significant or not.

Table 2 suggests the following points: 1) The dispersion in lithium $E W \mathrm{~s}$ is real in both the $E W$ vs. $B-V$ and the $E W$ vs. $V-I$ diagrams for stars in the color ranges $\mathrm{B}$ $\left(0.75 \leq B-V_{0} \leq 1\right)$ and $\mathrm{C}\left(B-V_{0}>1\right)$. As already known from previous studies, the dispersion in Li $E W$ s is instead not significant for earlier-type stars; 2) Our quantitative analysis supports points ii) and iii) above: namely, the dispersion in the $E W(\mathrm{~K})$ vs. $B-V$ diagram for stars with $B-V_{0}$ in the range $0.75-1$ is significant, while the dispersion is not significant for later and earlier-type stars. The dispersion is also not significant when considering $V-$ $I$ colors. Note that, as just mentioned in point 1 ), stars with $B-V_{0}>1$ do show a star-to-star scatter in Li $E W \mathrm{~s}$; 3) The $E W(\mathrm{Ca})$ vs. color diagrams are not characterized by a significant scatter; we get probabilities larger than $99.9 \%$ that the observed dispersion occurs by chance. As a final remark, we note that the reduced $\chi^{2}$ values obtained for the three lines and, most important, the probabilities that the observed dispersions are real, show a very weak dependence on the choice of the degree of the fit, i.e., a first order, linear fit provides similar results than a second order fit (see also Figs. 1-3). In other words, our results (and in particular the significance of the dispersion) do not appear to depend on the fitting procedure.

\subsection{Potassium excess vs. activity for IC 2602 and the Pleiades}

In the previous section we have shown that late- $\mathrm{G}$ and early-K IC 2602 stars are characterized by a dispersion in potassium $E W \mathrm{~s}$, at least when considering the $E W(\mathrm{~K})$ vs. $B-V$ distribution. The next issue is whether such a 

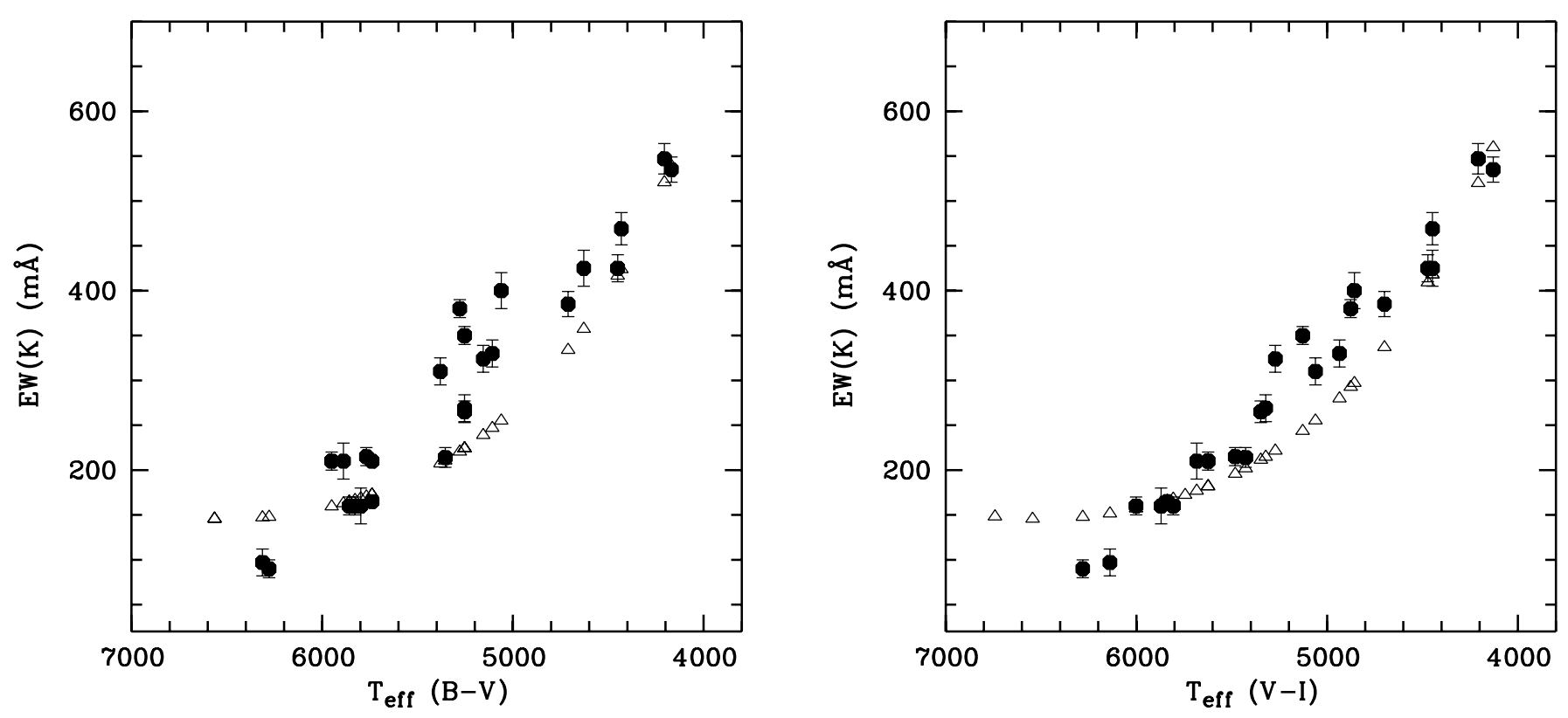

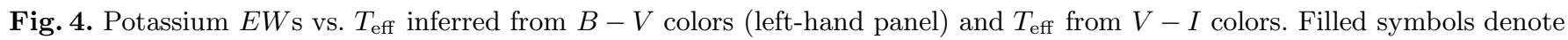
measured $E W \mathrm{~s}$ for our sample stars, while open symbols indicate predicted $E W \mathrm{~s}$ using the calibration of Tripicchio et al. (1999) (see text).

dispersion is caused by activity and, in particular, whether stars with different activity levels show any excess/deficit in their potassium $E W \mathrm{~s}$.

To address this question we computed for each star a predicted potassium $E W$ based on the empirical $E W(\mathrm{~K})$ vs. $T_{\text {eff }}$ relationship found by Tripicchio et al. (1999) for low activity field stars. More specifically, the predicted $E W$ s of the potassium line were determined using Eq. (1) in Tripicchio et al. (1999) and the $a_{i}$ coefficients for dwarf stars. Under the reasonable assumption that our cluster stars have a similar potassium abundance than field stars, differential potassium $E W$ s were then calculated as:

$\Delta E W=\left(E W_{\text {obs. }}-E W_{\text {pred. }}\right) / E W_{\text {obs. }}$,

where $E W_{\text {obs. }}$ and $E W_{\text {pred. }}$ are the observed and predicted values of the $E W$, respectively. Effective temperatures (and thus differential $E W \mathrm{~s}$ ) were derived based on both $B-V$ and $V-I$ colors. $T_{\text {eff }}(B-V)$ were estimated using a similar $T_{\text {eff }}$ vs. $B-V$ calibration than Tripicchio et al. (1999), namely, that of Gray (1992). Since Gray (1992) does not provide a $T_{\text {eff }}$ vs. $V-I$ calibration, we estimated $T_{\text {eff }}(V-I)$ as follows: we first computed the difference $\Delta T_{\text {eff }}$ between $T_{\text {eff }}(B-V)$ from Gray calibration and $T_{\text {eff }}(B-V)$ from the calibration employed by $\mathrm{R} 01$. Then we fitted the relation between $\Delta T_{\text {eff }}$ and $T_{\text {eff }}(V-I)$ from R01 and computed $T_{\text {eff }}(V-I)_{\text {Gray }}=$ $T_{\text {eff }}(V-I)_{\mathrm{R} 01}+\Delta T_{\text {eff. }}$. Before presenting and discussing our results, we caution that our quantitative estimate of differential potassium $E W$ s or potassium excesses is based on the fit of Tripicchio et al. (1999) and its accuracy; the comparison of our measured $E W \mathrm{~s}$ with predicted $E W \mathrm{~s}$ from different calibrations may not necessarily lead to the same quantitative results.
In Fig. 4 we plot the predicted (open triangles) and observed (filled circles) $E W \mathrm{~s}$ as a function of $T_{\text {eff }}(B-V)$ (left-hand panel) and $T_{\text {eff }}(V-I)$ (right-hand panel). The figure indicates that a large fraction of our sample stars indeed have larger $E W$ s than predicted; stars with $T_{\text {eff }}$ between 5400 and $5000 \mathrm{~K}\left(0.75 \lesssim B-V_{0} \lesssim 0.95\right)$ exhibit the largest excesses in the $E W$ vs. $T_{\text {eff }}(B-V)$ diagram; however, part of the stars that show an $E W$ excess in the $E W$ vs. $T_{\text {eff }}(B-V)$ diagram, have an $E W$ more in agreement with the predicted value when considering the $E W$ vs. $T_{\text {eff }}(V-I)$ diagram.

In Fig. 5 we plot the differential potassium $E W$ s vs. $\log L_{\mathrm{X}} / L_{\mathrm{bol}}$, the ratio of the X-ray over bolometric luminosity. $L_{\mathrm{X}} / L_{\text {bol }}$ values were retrieved from Stauffer et al. (1997) and are used here as activity tracers. If we exclude the two datapoints with the lowest differential equivalent widths ${ }^{1}$, a correlation between $\log L_{\mathrm{X}} / L_{\text {bol }}$ values and differential $E W \mathrm{~s}$ is evident in both panels. Although a oneto-one relationship between the two quantities cannot be claimed and for each $\log L_{\mathrm{X}} / L_{\mathrm{bol}}$ value a certain amount of scatter in $\triangle E W$ is present, stars with larger activity generally have larger $\Delta E W$ values, or larger excesses in $E W$. We computed the one-sided correlation coefficients finding that the correlations in both panels are significant at a confidence level larger than 99.99 (i.e., $>5 \sigma$ ), quantitatively confirming that more active stars tend to have larger $\mathrm{K}$ I $E W \mathrm{~s}$. We note that whereas a relationship between activity and $E W$ excess may be present also for

1 These datapoints correspond to the two warmest stars in the sample; they have rather weak potassium $E W$ s and we cannot exclude that their large deficit in $E W$ is due to measurement errors. 

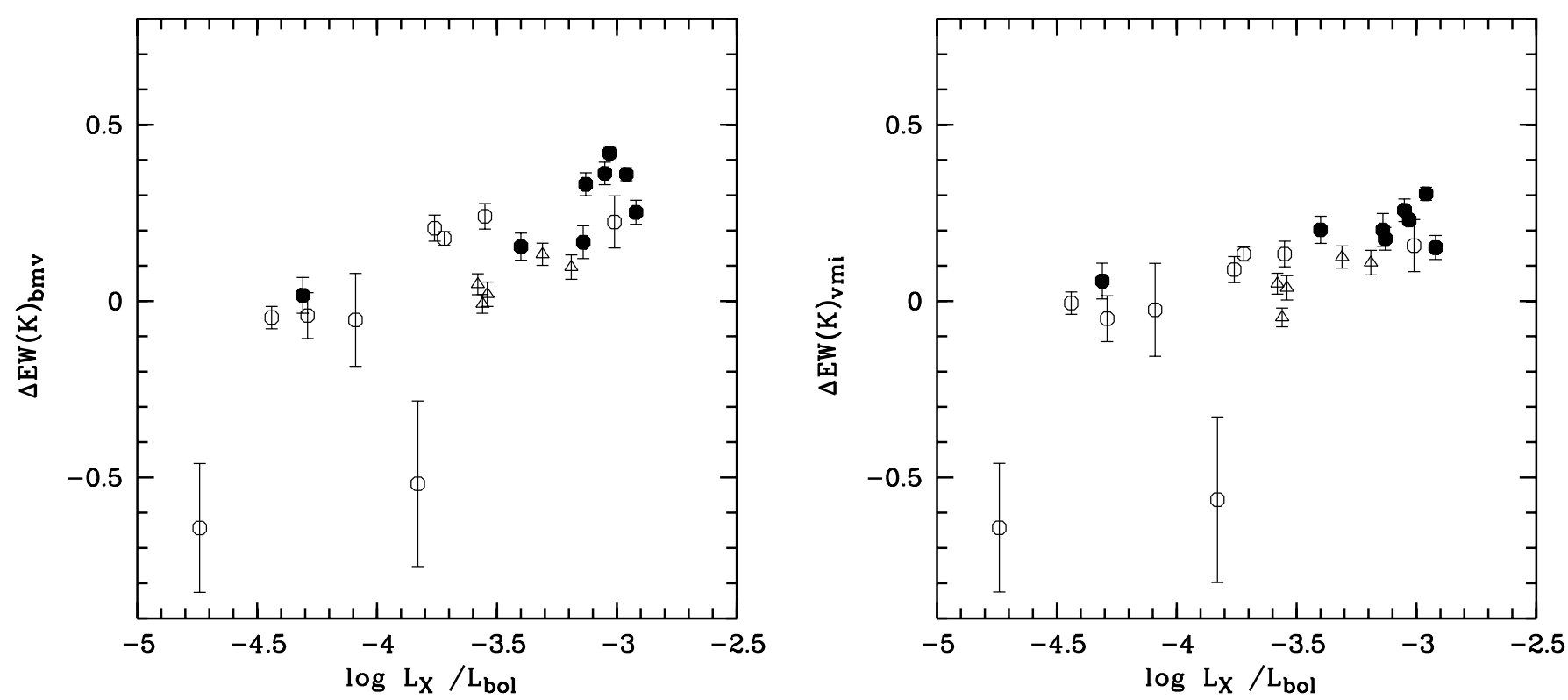

Fig. 5. Differential potassium $E W \mathrm{~s}\left(\Delta E W=\left(E W_{\mathrm{obs}}-E W_{\text {pred }}\right) / E W_{\text {obs }}\right)$ are plotted as a function of the logarithm of the ratio of X-ray over bolometric luminosity, used as an activity tracer. Left- and right-hand panels show differential $E W$ s computed with respect to predicted $E W$ s based on $T_{\text {eff }}(B-V)$ and $T_{\text {eff }}(V-I)$, respectively. Open circles denote stars with $B-V_{0}<0.75$, filled circles stars with $0.75 \leq B-V_{0} \leq 1$, and open triangles stars with $B-V_{0}>1$. Note that $\log L_{\mathrm{X}} / L_{\mathrm{bol}}$ values are not available for all our sample stars.
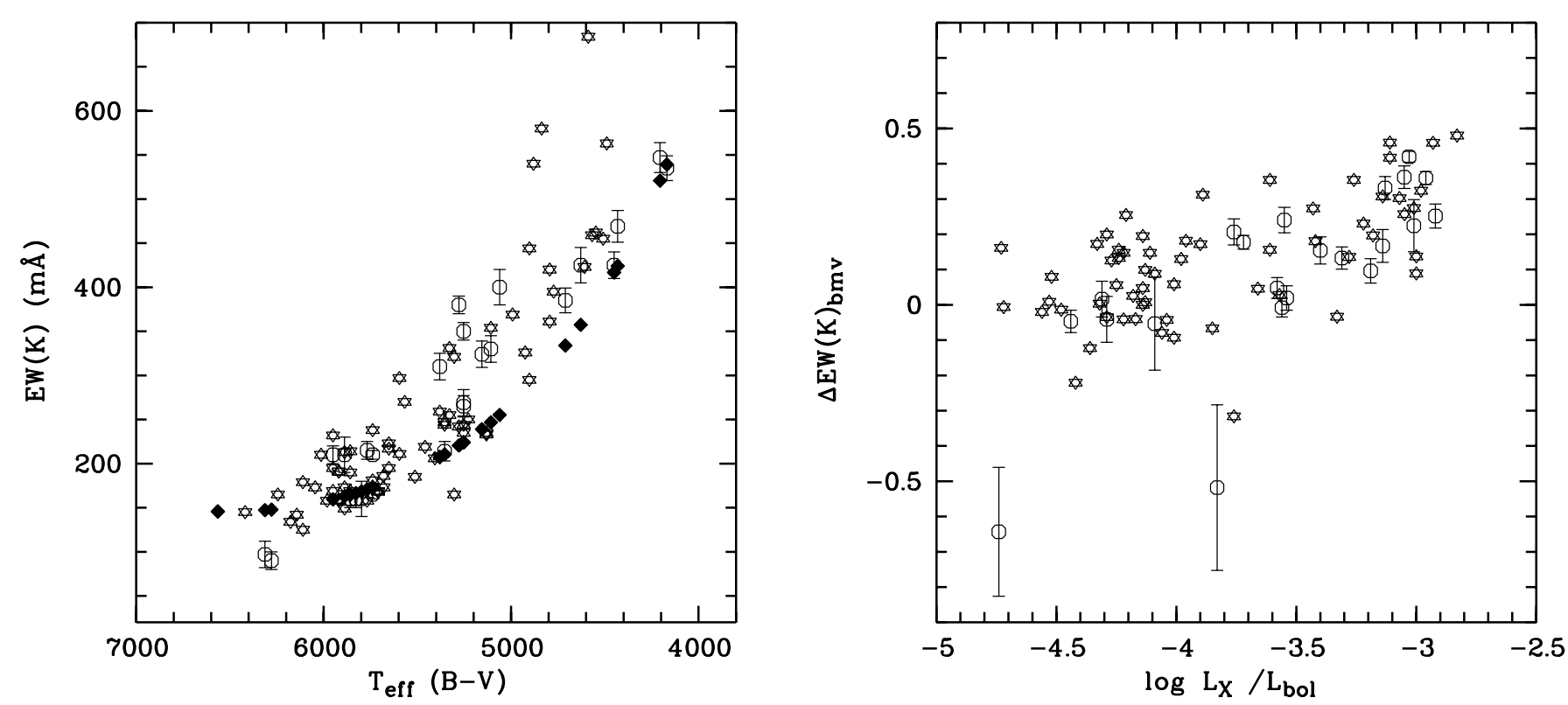

Fig. 6. Left-hand panel: potassium $E W \mathrm{~s}$ vs. $T_{\text {eff }}(B-V)$. Filled symbols indicate predicted $E W \mathrm{~s}$ based on Tripicchio et al. (1999), while open circles and stars denote the observed $E W$ s for IC 2602 and the Pleiades, respectively. Right-hand panel: $B-V$ based differential potassium $E W \mathrm{~s}$ as a function of $\log L_{\mathrm{X}} / L_{\mathrm{bol}}$ for our sample stars (open circles) and the Pleiades (open stars).

our sample stars with $B-V_{0} \geq 1$, these stars cover a rather narrow range of $\log L_{\mathrm{X}} / L_{\mathrm{bol}}$ values and, consequently, a narrow range of differential $E W \mathrm{~s}$; finally, for a given $\log L_{\mathrm{X}} / L_{\mathrm{bol}}$ or activity level, several stars have larger differential $E W \mathrm{~s}$ when considering the predicted $E W$ based on $T_{\text {eff }}(B-V)$ than those based on $T_{\text {eff }}(V-I)$.
Figures $6 \mathrm{a}$ and $6 \mathrm{~b}$ are similar to the left-hand panels of Figs. 4 and 5, but our sample stars are compared to the Pleiades. Potassium EWs for this cluster were retrieved from Soderblom et al. (1993a), while $\log L_{\mathrm{X}} / L_{\mathrm{bol}}$ values were taken from Stauffer et al. (1994) and Micela et al. (1996). As for our sample stars, effective 
temperatures were inferred using the $T_{\text {eff }}$ vs. $B-V$ calibration of Gray (1992). The figure clearly shows that the two clusters behave very similarly; in particular Fig. $6 \mathrm{~b}$ indicates that, above $\log L_{\mathrm{X}} / L_{\mathrm{bol}} \sim-4$, stars with similar activity levels have similar excesses in potassium $E W$ s. Low activity Pleiades stars instead exhibit a scatter in differential $E W$ s, while all IC 2602 members with $\log L_{\mathrm{X}} / L_{\text {bol }}<-4$ have $\Delta E W(\mathrm{~K}) \sim 0$. The rather small number of low-activity IC 2602 members and the lack of available errors for the potassium $E W \mathrm{~s}$ of the Pleiades, do not allow us to ascertain whether this difference is significant or not. In any case, as for IC 2602, we find that the correlation between differential $E W \mathrm{~s}$ and $\log L_{\mathrm{X}} / L_{\mathrm{bol}}$ values for the Pleiades is significant at $>99 \%$ confidence level. Note that, whereas a relationship between differential $E W \mathrm{~s}$ and activity for the Pleiades was already found by King et al. (2000), we computed $E W$ excesses with respect to inactive field stars, while their $E W$ excesses (deficits) referred to a mean trend in the $E W$ vs. $T_{\text {eff }}$ diagrams.

\section{Discussion}

What do the results presented in the previous section allow us to conclude? First, the findings of King et al. (2000) and of similar papers for the Pleiades seem to hold also for the younger IC 2602: a dispersion in potassium $E W \mathrm{~s}$ is detected. In addition, active stars in IC 2602 and the Pleiades show potassium $E W \mathrm{~s}$ in excess of those of inactive field stars with similar colors; a statistically significant correlation between $E W$ excess and activity is found for both clusters. These results support the hypothesis that the appearance of the alkali $E W$ vs. color distributions in young clusters is affected by activity which alters the formation of the resonance lines. Activity seems to affect stars at all colors, although different dispersions in the activity levels reflect into different spreads in the potassium $E W$ for stars in different color ranges.

Second, no significant dispersion in the $E W(\mathrm{Ca})$ vs. color diagrams is found: so far no study has tried to model how activity and the presence of surface inhomogeneities would affect the observed Ca I vs. color diagrams. Activity is not thought to affect the formation of the Ca I line itself; however, large and cool spots, besides affecting the observed $E W$ s of the alkali atoms, are also predicted to alter stellar colors (Stuik et al. 1997; Barrado y Navascués et al. 2001). The Ca $E W$ vs. color distributions can thus be used to put some constraints on the characteristics of the spots. If $B-V$ colors of our cluster stars were significantly altered by the presence of surface inhomogeneities, a dispersion should also be observed in the $E W(\mathrm{Ca})$ vs. color diagrams, since stars with the same intrinsic color, but different activity levels would have the same Ca $E W$, but different observed colors. Viceversa, at a given observed color one would find stars with different intrinsic colors and thus Ca $E W$ s. More specifically, the absence of a detectable scatter in the $E W$ of the Ca line suggests that the characteristics of surface inhomogeneities in our sam- ple stars should be such that stellar colors are not greatly altered. Given the $E W(\mathrm{Ca})$ vs. $B-V$ relationship for our sample stars, $\Delta B-V$ larger than 0.1 dex are required to have $\Delta E W(\mathrm{Ca})>2 \sigma(E W(\mathrm{Ca}))$ and hence a detectable spread in Ca $E W$ s.

Figures 1b and 3a of Barrado y Navascués et al. (2001) show the predicted variations in $B-V$ colors and potassium equivalent widths vs. spot coverage for different spot temperatures. Figure $1 b$ indicates that in order to have variations in $B-V$ colors $\Delta B-V$ values larger than $\sim 0.1$ dex, filling factors larger than $40 \%$ (or more, depending on the $\Delta T$ between the spot and the quiet photosphere) are needed. On the other hand, differential $E W \mathrm{~s}$ of our sample stars can be as large as $\sim 0.4$ dex (see Fig. 5 ): the comparison with Fig. 3a of Barrado y Navascués et al. (2001) shows that, assuming spot coverages below $\sim 40 \%$, these values can be obtained for $\Delta T$ between the spot and the quiet photosphere of the order of $\sim 1000 \mathrm{~K}$. In other words, the whole range of differential potassium EW s measured among IC 2602 stars is consistent with the predictions of Barrado y Navascués et al. (2001), provided that our sample stars are covered by cool enough spots.

Third, we find that the $E W(\mathrm{~K})$ vs. $V-I$ diagram is not characterized by a significant scatter (neither for stars in different color ranges, nor when considering the whole range); in addition, differential $E W$ s from a $V-$ $I$ based analysis are somewhat smaller than those from a $B-V$ based analysis. This means that most of our sample stars appear redder/cooler when considering $V-I$ colors. We do not have a definitive explanation for this finding, but can attempt two different hypothesis: namely, either $B-V$ colors are not correct and thus they are not good temperature indicators and $V-I$ colors should always be used (but in this case it would be hard to explain the rather tight $E W(\mathrm{Ca})$ vs. $B-V$ relationship); or $V-I$ colors are somewhat more affected by spots than $B-V$ colors; if the increase in $E W$ is accompanied by a shift in color, an active star with a given color and $E W$ may move, in the $E W(\mathrm{~K})$ vs. color plane, close to a cooler less active star with an intrinsically redder color and larger $E W$. Although Barrado y Navascués et al. (2001) and similar studies have not modeled the effect of spots on $V-I$ colors it is reasonable to think that cool enough spots would affect more $V-I$ than $B-V$ colors.

\section{Conclusion: Is the spread in lithium due to activity?}

We have measured the $E W$ of the $\mathrm{K}$ I line in a sample of late-type members of IC 2602. Our study confirms the results of previous papers based on the Pleiades cluster, but, at the same time, adds new pieces of information into the issue of the scatter in $\mathrm{Li} / \mathrm{K}$ observed among late-type stars in young clusters. The results and discussion presented in the previous sections support the idea that the potassium $E W$ vs. color diagrams of IC 2602 stars are affected by activity. Based on a statistical analysis, a star-to-star scatter 
in potassium $E W \mathrm{~s}$ is detected in the $E W$ vs. $B-V$ diagram of late- $\mathrm{G}$ /early-K-type stars. In addition, the most active IC 2602 (and Pleiades) stars show EW excesses with respect to inactive field stars; differential $E W \mathrm{~s}$ as large as 0.4 dex are measured, with more active stars generally having larger $E W$ excesses. More specifically, a significant correlation between potassium excess and $\log L_{\mathrm{X}} / L_{\text {bol }}$ ratios is found for both IC 2602 and the Pleiades. The dispersion in the $E W(\mathrm{~K})$ vs. $V-I$ diagram is not significant and we suggest that this may be due to the different effect that cool spots have on $B-V$ and $V-I$ colors. We also find that stars later than $B-V_{0} \sim 1$ do not show a significant dispersion in $E W(\mathrm{~K})$, although they do show $E W(\mathrm{~K})$ excesses. We believe that the reason for this is the narrow range in activity levels covered by late-type cluster members.

The question remains whether the observed scatter in Li EWS can then be fully explained as due to activity. To answer this question the same type of analysis that we presented for potassium should in principle be carried out also for lithium; namely, the Li $E W$ s of cluster stars should be compared with those of a sample of inactive field stars in order to look for $E W(\mathrm{Li})$ excesses. However, due to the age-magnetic activity inverse relationship, inactive field stars are most likely old and have hence undergone a significant amount of Li depletion; their intrinsic Li abundance is presumably lower than that of IC 2602 members and the comparison would be meaningless. The comparison of measured $E W$ s in IC 2602 with model predictions for the age of the cluster would not work either: standard models depend on the adopted assumptions on e.g. convective treatment and atmospheric opacities, and different groups make different quantitative predictions on Li depletion as a function of mass; therefore the comparison, and in particular any Li excess/deficit that we would find, would depend on the particular choice of the model.

The most secure conclusion that can be drawn from the present study is that the scatter in lithium among stars later than $B-V_{0} \sim 1$ cannot be explained by activity only. The detection of $E W(\mathrm{~K})$ excesses among late-K cluster members suggests that the formation of the $\mathrm{K} I$ feature in these stars is affected by activity; however, as we have discussed, these stars (at least those included in the present sample) are characterized by similar activity levels and thus show little scatter in their potassium $E W$ s. The same should hold for the Li line, in contradiction with the large observed scatter in $\mathrm{Li}$ among our sample stars with $B-V_{0}>1$. In other words, although the Li $E W$ s of the coolest stars in our sample are most likely affected by the presence of surface inhomogeneities (since potassium $E W \mathrm{~s}$ are affected), the lack of scatter in potassium $E W \mathrm{~s}$ among these stars suggests that the ultimate reason for their dispersion in $\mathrm{Li}$ abundances is a different amount of Li destruction.

As for earlier-type stars, at this stage we cannot answer the question whether their scatter in $\mathrm{Li} E W \mathrm{~s}$ is due to a scatter in abundances, and hence different amounts of $\mathrm{Li}$ depletion, or is instead completely due to activity.
Certainly, it is at least in part caused by activity. If we focus on a narrow color range (e.g. $0.79 \lesssim B-V_{0} \lesssim 0.84$ ), stars with larger potassium $E W$ excesses tend to have larger Li $E W$ s; since within 0.05 dex difference in $B-V$ (or $\sim 100 \mathrm{~K}$ interval in $T_{\text {eff }}$ ) a large difference in Li abundance is not expected, this provides an hint that the difference in $\mathrm{Li} E W_{\mathrm{S}}$ is really due to a difference in activity only. Unfortunately, there are only five stars in this color range, which does not allow us to regard this conclusion as definitive.

Most obviously, further investigations on this topic should be carried out, both on theoretical and observational grounds. The potassium line should be measured in additional clusters. Additional photometry, and in particular photometric monitoring and/or, as mentioned by Jeffries (1999), Doppler imaging of cluster stars, which would allow constraining the characteristics of spots and plages and their timescales, should also be performed. If possible, simultaneous monitoring of the alkali lines $E W \mathrm{~s}$ (including also the Na I $5896 \AA$ feature) should be obtained. At the same time, additional modeling should be carried out.

Acknowledgements. I thank an anonymous referee for her/his very useful comments and suggestions. I am grateful to John Stauffer for making available to me CTIO spectra of three stars in the sample.

\section{References}

Balachandran, S., Lambert, D. L., \& Stauffer, J. R. 1996, ApJ, 470, 1243

Barrado y Navascués, D., García López, R. J., Severino, G., \& Gomez, M. T. 2001, A\&A, 371, 652

Butler, R. P., Cohen, R. D., Duncan, D. K., \& Marcy, G. W. 1987, ApJ, 319, L19

Duncan, D. K., \& Jones, B. F. 1983, ApJ, 271, 663

García López, R. J., Rebolo, R., \& Martín, E. L. 1994, A\&A, 282,518

Gray, D. 1992, The observation and analysis of stellar photospheres, 2nd edition (Cambridge University Press)

James, D. J., \& Jeffries, R. D. 1997, MNRAS, 292, 252

James, D. J., Collier Cameron, A., Barnes, J. R., \& Jeffries, R. D. 2000, in Stellar Clusters and Associations: Convection, Rotation, and Dynamos, ed. R. Pallavicini, G. Micela, \& S. Sciortino, ASP Conf. Ser. 198, 277

Jeffries, R. D. 1999, MNRAS, 309, 189

Jeffries, R. D. 2000, in Stellar Clusters and Associations: Convection, Rotation, and Dynamos, ed. R. Pallavicini, G. Micela, \& S. Sciortino, ASP Conf. Ser. 198, 245

Jones, B. F., Shetrone, M., Fisher, D., \& Soderblom, D. R. 1996, AJ, 112, 186

Jones, B. F., Fischer, D., Shetrone, M., \& Soderblom, D. R. 1997, AJ, 114, 352

King, J. R., Krishnamurthi, A., \& Pinsonneault, M. H. 2000, AJ, 119, 859

Martín, E. L., \& Claret, A. 1996, A\&A, 306, 408

Martín, E. L., \& Montes, D. 1997, A\&A, 318, 805

Micela, G., Sciortino, S., Kashyap, V., Harnden, F. R., Jr., \& Rosner, R. 1996, ApJS, 102, 75

Patten, B. M., \& Simon, T. 1996, ApJS, 106, 489 
Pinsonneault, M. S. 1997, ARA\&A, 35, 557

Prosser, C. F., Randich, S., \& Stauffer, J. R. 1996, AJ, 112, 649

Randich, S., Schmitt, J. H. M. M., Prosser, C. F., \& Stauffer, J. R. 1995, A\&A, 300, 134

Randich, S., Aharpour, N., Pallavicini, R., Prosser, C. F., \& Stauffer, J. R. 1997, A\&A, 323, 86

Randich, S., Martín, E. L., García López, R., \& Pallavicini, R. 1998, A\&A, 333, 591

Randich, S., Pallavicini, R., \& Mermilliod, J.-C. 2000, IAU Symp. 198, ed. M. Spite, \& L. da Silva, 287

Randich, S., Pallavicini, R., Meola, G., Stauffer, R. J., \& Balachandran, S. 2001, A\&A, 372, 862
Soderblom, D. R., Jones, B. F., Balachandran, S., et al. 1993a, AJ, 106, 1059

Soderblom, D. R., Stauffer, J. R., Hudon, J. D., \& Jones, B. F. 1993b, ApJS, 85, 315

Stauffer, J. R., Caillault, J.-P., Gagne, M., Prosser, C. F., \& Hartmann, L. W. 1994, ApJS, 91, 625

Stauffer, J. R., Hartmann, L. W., Prosser, C. F., Randich, S., \& Balachandran, S. 1997, ApJ, 479, 776

Stuik, R., Bruls, J. H. M. J., \& Rutten, R. J. 1997, A\&A, 322, 911

Whiteoak, J. B. 1961, MNRAS, 123, 245

Tripicchio, A., Gomez, M. T., Severino, G., et al. 1999, A\&A, 345,915 